\title{
Programs' Efficacy to Develop Employable Skills for People With Functional Diversity: A Meta-Analysis
}

\author{
Carolina Gonzálvez ${ }^{1 \star}$, Fernando Marhuenda-Fluixà ${ }^{2}$ \\ ${ }^{1}$ Departament of Developmental Psychology and Teaching, University of Alicante, \\ Ctra. San Vicente, S/N. 03690, San Vicent del Raspeig, Spain \\ ${ }^{2}$ Department of Didactics and School Organization, University of Valencia, Avda. \\ Blasco Ibáñez 30, 46010, València, Spain
}

Received: 23 November 2020, Accepted: 15 September 2021

\begin{abstract}
Purpose: Promoting the labour integration of people with functional diversity is a key element to achieve their social inclusion. This meta-analysis aims to examine the effectiveness of experimental programs in developing employable skills for people with disabilities.

Methods: Literature searches up to June 2019 were conducted in four databases (Web of Science, Scopus, PsycINFO and ERIC). Studies that met the following criteria were selected: (1) The program should develop employable skills; (2) the participants should be people with functional diversity; (3) the study should have a design with an experimental group and a control group as well as pretest and posttest measurements; (4) the study had to provide enough data to calculate the effect sizes; and (5) the study had to be written in English or Spanish. 67 independent studies met the selection criteria, among 14 articles published between 1998 and 2019.

Results: The results revealed mean effect sizes in favour of the experimental group for the set of all studies according to data reported by people with functional diversity, as well as according their relatives and teachers. The two dimensions of the programs with a significant effect size in favour of the experimental group were interview skills and career
\end{abstract}

*Corresponding author: carolina.gonzalvez@ua.es 
planning. Furthermore, it was found that the programs showed a higher degree of effectiveness in groups formed only by people with intellectual disabilities, with a lower educational level, whose duration ranged from six to twelve months. This was particularly the case with participants from Spain and Australia.

Conclusion: Promoting the labour insertion of people with disability is a key element to achieve their social inclusion. Programs that support and develop employability and that are conducted upon experimental conditions do have a positive impact upon young people with functional diversity. Upon the results, we discuss practical implications for integrating disabled persons into the labour market.

Keywords: Employability, Transition From Education and Training to Employment, People With Disability, Career Planning, Vocational Education and Training, VET

\section{Introduction}

Along history, the notion of disability and the legislation that addresses it have evolved and have conditioned the actions and support provided to people with functional diversity. This last concept, considered less offensive and more appropriate these days, refers specifically to people who perform certain functions differently than the average population so they are different than the statistical norm due to some reason (Senent-Ramos, 2020), even though many academic and policy documents use disability, like the Convention on the Rights of Persons with Disabilities (United Nations, 2006) or the European disability strategy 2010-2020 (European Union, 2010). In the past decades, there has been an evolution towards conceptual models that define disability in rather inclusive ways; a shift has been identified from a traditional individualist segregation model, where religious or magical explanations are provided, towards a social model based upon the relevance of personal autonomy (López-Bastías, 2019). This approach contributes to a practice that acknowledges the principle of equal opportunities, and this favors self-determination of people with disability. Accordingly, in recent years there has been an increase in actions and initiatives oriented towards diminishing inequalities that people with functional diversity face both in personal and professional domains. Nevertheless, despite the update of the notion of disability has implied a proliferation of legal and institutional measures regarding disability, these approaches are not exclusive and they are able to coexist in a given time, in such a way that inequalities remain.

According to the statistics of the United Nations, $82 \%$ of people with disability who live in developing countries are below poverty threshold (Martínez-Ríos, 2011). In Spain, according to the National Institute of Statistics (2019) employment rate among people with disabilities was $25.9 \%$ in 2017 , while people without disabilities doubled that number, reaching $64.4 \%$, 
similarly to previous years. Therefore, restrictions in access to employment have implied an impulse for legal measures and other actions that attempt to reduce such inequalities.

The United Nations Convention on the Rights of People with Disabilities (United Nations, 2006) has established a new policy framework to strengthen their rights and liberties, whatever their condition. Furthermore, this organization suggested in 2015 the Agenda 2030, an action plan with the aim to achieve the world commitment in favor of people, the planet and prosperity. Among its aims, the acknowledgement and protection of rights of people with disabilities (Madans et al., 2017).

Nevertheless, despite progress regarding equal opportunities, no discrimination and universal accessibility of people with disabilities; there are yet huge differences in employment indexes of people with disabilities and the rest of the active population, as well as prejudice and discrimination in work relations (Goldman et al., 2006; Schur et al., 2009). There are also reports that show that recession and economic crisis have a negative impact upon this people, with increasing wage differences with people without disabilities (Eurofound, 2013; National Institute of Statistics, 2017) resulting in damages upon health and welfare.

Active participation in the labor market is one of the keys in quality of life of the population and this is also the case for people with functional diversity (Novo-Corti, 2018), and this is more the case in times of growing precariousness. Access to decent work is crucial in order to achieve social inclusion. Labor integration of people with functional diversity has been and is an aim of social policies and employment for international organizations and an increasing number of countries adopt them (Douvitsa, 2020; Fici, 2020; Maffiioletti \& Sato, 2020; Meira, 2020; Salinas \& Marhuenda, 2020). The social economy and Third Sector Organisations are playing a major role here (Bengoetxea \& Fajardo, 2020; Marhuenda, 2021). This requires not only technical competence, but also social and personal abilities that allow workers to be employable or, in terms of Eraut (2004), to be competent in different domains or learning trajectories (job performance, role performance, working in teams, understanding and awareness or personal development among others). Findings about these possibilities with vulnerable groups have already been published (Chisvert et al., 2018; Marhuenda, 2018). In this sense, learning to work and becoming a worker are processes closely connected to becoming an adult and a citizen too, not just in individual terms, but also in societal ones; as it is the status of worker what provides acknowledgement for the status of citizen and of autonomous life, one free of dependency support provided by the administration, the families or volunteering practices.

In this regard, transition is a crucial dimension in our research, and it becomes the focus of our search question too: Transition programs are holistic as they intend to provide different supports (education, housing, health, counselling and leisure among them), as Casal (1996), Casal et al. (2015) and Merino $(2007,2019)$ have shown. Transition processes have become a stage of its own (Walther, 2006), beyond their instrumentality in getting a job, and it is in this context that employability gains relevance too: As a matter of fact, being employable 
does not guarantee getting a job, but it is an indicator of readiness. Employability has been discussed in its relation to work and education (Gonon et al., 2008) as well as specifically focused upon people living in vulnerable circumstances (long term unemployed, people at risk of social exclusion, people with disabilities) in its individual and contextual features (Córdoba et al., 2013; Llinares et al., 2016). Transition programs try to foster employability rather than finding a job in themselves, and they do so by improving personal and social skills of the individuals, as well as their wellbeing along the process. This involves also addressing issues other than work-related ones (Hofmann et al., 2021). At this point, dealing with personal expectations, career guidance and planning, social skills to interact with other adults and self-confidence and self-knowledge are important dimensions in these programs. Therefore, occupational training is relevant, as well as the qualification level, but given limitations introduced by disabilities, the kind of disabilities have an impact upon expectations and ability to learn (Badiola et al., 2014; Elorriaga et al., 2019; International Labour Organization, 2016). Therefore, the kind of disability, educational background and duration of the program are dimensions that could have an impact upon intervention, as the country is also expected to do insofar there are specific policies, regulations and protective measures that have to be considered. Therefore, transition programs for vulnerable people often try to facilitate becoming an adult and an autonomous citizen, not just a worker: Nowadays, training for the labour market consists also of preparation for unemployment, even to increasingly precarious working conditions.

Much research has focused upon the study of transition into adult life of people with disability, without having the desired effect to improve social and labor inclusion (Dyke et al., 2013). Therefore, Pallisera et al. (2018) took the effort to identify, through focus groups with people with disabilities, their families and professionals in the fields of education and social work, the main obstacles that they face. Among these, the need to offer them a more flexible curriculum fostering their labor inclusion and the need to improve career guidance, particularly at the end of Secondary Education. It is therefore worth paying attention to training addressed to improve the employability of people with disability. Facilitating labor insertion of people with functional diversity through training opportunities, information and practice of personal, social and technical skills to have access into the world of work is crucial in this regard. Vocational education aimed at accredited qualification is key to equip youth with functional diversity to enter the labor market (Gilson et al., 2017).

Several systematic reviews have rigorously explained the 'state of the art' about this issue. Gilson et al. (2017) provided a systematic review through the analysis of 56 studies about different training methods for training employment skills among people with disabilities. They identified 21 such methods, among which four stranded out: Assessment of performance feedback, device-assisted instruction, response prompting and community-based instruction. Engelbrecht et al. (2017) reviewed 99 documents on transition programs into work for people with disabilities highlighting the need for further research. Several systematic reviews on peo- 
ple with autistic spectrum disorder (ASD) pay attention to the features and results of actions based upon employment programs, transition into work and occupational activities (Hedley et al., 2017; Lee et al., 2018; Taylor et al., 2012; Walsh et al., 2017). Among these, Lee et al. (2018) point to practice within these employment programs focused not only in fostering occupational skills and improving employability results, but which also provide collateral benefits improving mental health, self-confidence or time-management, among others.

Despite systematic reviews show results indicating scientific standards, objectivity, systematization and replicability (Botella \& Sánchez-Meca, 2015), it is not possible to draw conclusions based upon the measurement of effectiveness. In order to do so, meta-analytical studies allow to apply statistical techniques to integrate results in a set of empirical studies with the aim to establish the efficacy of certain programs and interventions.

Park et al. (2016) developed a meta-analysis with seven random controlled trials which analyzed the effect of training students with disabilities in social skills related to employment. Results pointed to a moderated effect size, and they found a larger effect in those studies applying direct instruction, particularly in cases with ASD, emotional or behavioral disorder and intellectual disability. Among the limitation of this research, they analyzed the isolated effect of social skills, without considering other relevant skills in access to the labor market. More recently, Alson et al. (2019), conducted a survey among managers and human resource officers, and they identified that the skills most demanded among people with disability are communication, teamwork, analysis and use of computer applications. Therefore, skills expected to be found among people with functional diversity when accessing employment are wider than mere social skills. Nonetheless, there have been no meta-analysis that analyze the effectiveness of employment programs considering skills in a wider sense.

Upon review of scientific literature, we have detected and increase in the number of studies conducted in recent years on access to employment of people with functional diversity. We are also conscious of the different measures taken to improve the awareness and engagement along the world to support the rights of people with disabilities. Nevertheless, employability indexes of people with functional diversity do not reach the expectations that neither programs nor research have cherished. Before this limitation, it seems adequate and timely to conduct a meta-analysis to allow identification of how effective prove employment programs for people with functional diversity and which are the conditions they embed that increase the success of such measures.

Therefore, our aim is to conduct a meta-analysis to assess the efficacy of employment programs addressed to young people with functional diversity. The research question we attempt to answer in our work is the following: What is the effectiveness of transition programs under experimental conditions that develop employability skills for young people with functional diversity; where effectiveness consists of a statistically significant larger size effect in the experimental groups in relation to the control groups. 


\section{Methods}

\subsection{Selection Criteria}

Studies that met the following criteria were selected: (1) The program should develop employable skills; (2) the participants should be people with functional diversity; (3) the study should have a design with an experimental group and a control group as well as pretest and posttest measurements; (4) the study had to provide enough data to calculate the effect sizes; and (5) the study had to be written in English or Spanish.

\subsection{Data Sources and Search Strategy}

Studies were found using the Web of Science, SCOPUS, PsycINFO and ERIC databases for studies published in English or Spanish up to June 2019. The search strategy used was (program $^{\star}$ or intervent ${ }^{*}$ or train ${ }^{\star}$ ) AND transition AND (employment or job or work) AND $\left(\text { disab }^{*} \text { or autis }{ }^{\star}\right)^{1}$. No limitations in the search strategy were considered. In addition, other sources (e.g., google scholar and the references' list of the theoretical reviews) were used to rescue research that may not have been recovered from the afore mentioned databases.

Figure 1 presents the selection process followed by the PRISMA checklist. 3934 records were identified, of which 3048 did not simultaneously appear in all the databases used. From these studies, 2527 were excluded for not addressing the specified topic of research or for being written in languages such as Korean and Russian. Thus, 521 works were subject to the inclusion criteria, identifying a final sample of 14 studies that developed employable skills through programs for youth with functional diversity. The exhaustive reading of the articles allowed us to confirm the choice of all 14 papers given that they met all the inclusion criteria. Some of them included different measures of employable skills and other variables, so they were analyzed as independent meta-analyses. This study was developed in accordance with the PRISMA diagnostic test accuracy checklist that provides specific guidance for reporting of systematic reviews and meta-analysis (McInnes et al., 2018).

1 Following a reviewer's recommendation, we have checked in the four databases and stablishing the same time limit (June 2019, except in Scopus -through the year 2019-), to check whether including new keywords in the search strategy should lead us to include new articles in the meta-analysis because of meeting all the inclusion criteria. We tested the following search strategy (program* or intervent ${ }^{\star}$ or $\operatorname{train}^{\star}$ ) AND transition AND (employment or job or work or employability or "employment skills") AND (disab* or autis* or "special needs") and the results found were: Web of Science 1667 results (207 more), Scopus 1000 results all the year 2019 (86 more), ERIC 1209 results (334 more) and PsycINFO 1399 results ( 720 more). Although the number of studies has increased, none of them meets the selection criteria reporting enough data to calculate the effect size and be implemented with an experimental group and a control group as well as offering pre-test and post-test measurements. 

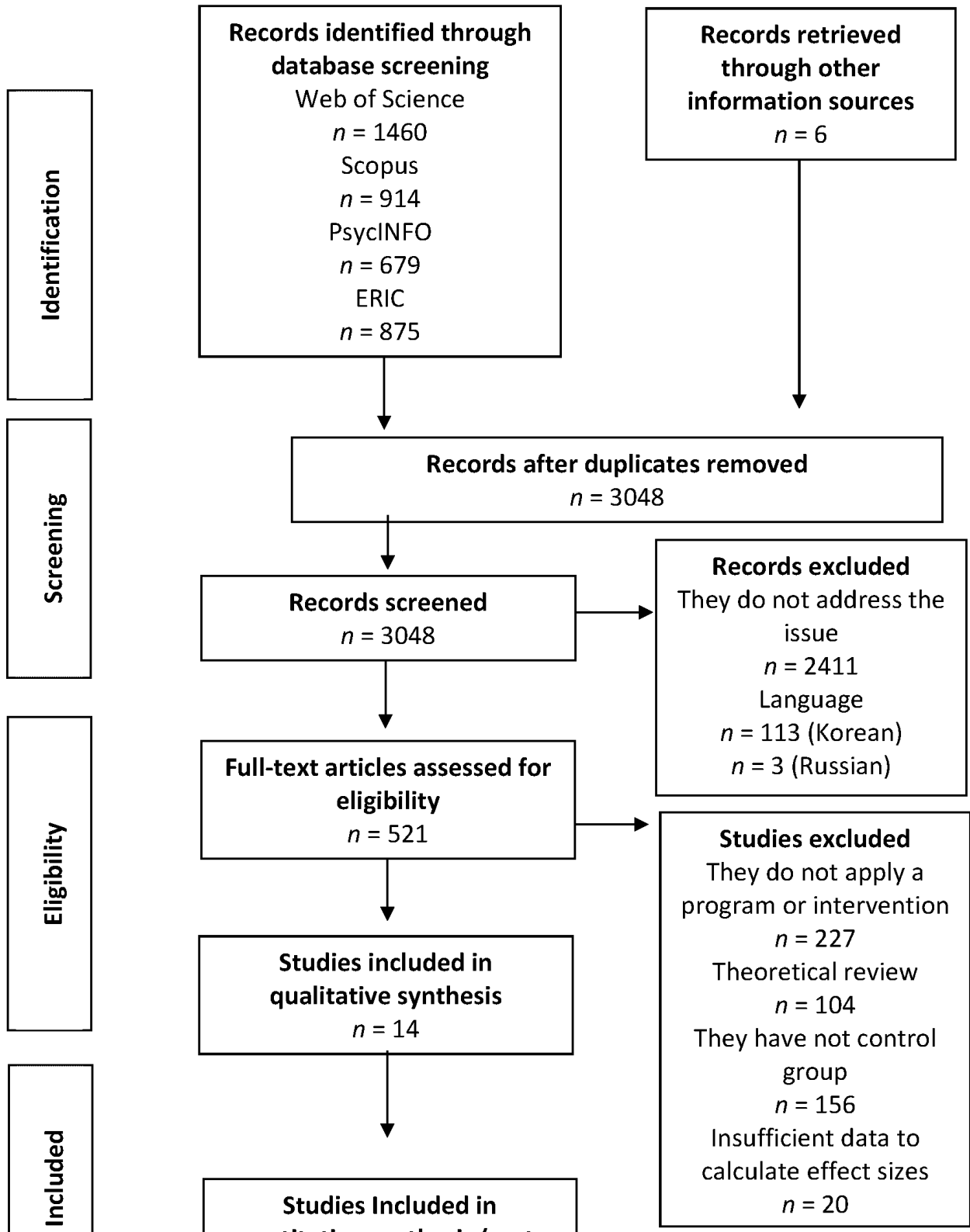
quantitative synthesis (metaanalysis) $n=14$

Figure 1: PRISMA Flow Diagram for Study Selection 


\subsection{Coding Moderator Variables}

In order to analyze the heterogeneity between the results of the studies, the moderator variables that could be related with the effect sizes were coded. The moderator variables were classified according to Lipsey's classification (1994) as (a) participants' variables, that include personal characteristics of the samples (e.g. type of disability or disorder, the age range of the sample, the gender distribution, intelligence quotient); (b) treatment's variables, which are those that explain intervention characteristics (e.g. the magnitude of total duration, number of weekly hours of treatment (frequency), proportion of participants, use of the technology, teaching profile); (c) methodological characteristics, which have to do with the design of the research and the implementation of the empirical study (e.g. randomly group formation or not, type of control group (active versus non-active), number of participants in the control and experimental groups); and (d) extrinsic characteristics, which, in principle, should not relate to the scientific process, but that can be associated with the results (e.g. year of publication or country). The coding process was performed separately by two researchers in order to finally obtain a reliable and accurate code relationship.

\subsection{Statistical Analyses}

In this meta-analysis a random effect model was used. This statistical model is considered more appropriate because:

"Is more likely to fit the actual sampling distribution, does not impose a restriction of a common effect size, yields the identical results as the fixed-effect model in the absence of heterogeneity and allows the conclusions to be generalized to a wider array of situation." (Borenstein et al., 2010, p. 107)

Typified mean change difference, d, was chosen as a measure of effect size (Glass et al., 1981). Positive values of $\mathrm{d}$ reflected an improvement from pretest to posttest in the treated group. The statistical analysis of the effect sizes was based on the model proposed by Hedges and Olkin (1985), according to which each effect size is weighted based on the inverse of its variance, so that studies with higher sample sizes exert a greater specific weight in these analyses. The mean efficacy was calculated and the heterogeneity was assessed using the $Q$ test and the index $\mathrm{I}^{2}$ (Higgins \& Thompson, 2002). Statistical significance was set at $p<.05$ and significant heterogeneity was considered with the following values $p<.05$ and $\mathrm{I}^{2}>50 \%$. The heterogeneity will be explored through the influence of moderating variables. Statistical analyses were calculated with the RevMan5.3 program (2014). 


\section{Results}

\subsection{Descriptive Features of the Studies}

Table 1 provides the descriptive features of the studies included in this meta-analysis. Fourteen studies met all inclusion criteria, among which 67 independent studies were identified, given that most of this research analyzed data from more than a variable upon different evaluation tools. Research was published between 1998 and 2019, 85.7\% being published in the current decade.

Among features defining participants, age range varies between 12 and 55. Regarding sex, most of the studies entail mixed samples. Only one of the studies was conducted with only boys (Strickland et al., 2013) and there was also another study with only girls (Lindstrom et al., 2013). Seven out of the 14 reports were shaped by people with autistic spectrum disorder, one of them with people with intellectual disability, two in which participants had several difficulties and/or disabilities. Regarding intelligence quotient, while nine of the reports do not provide these data, in those which do so, most participants were above 70. Six of the reports do not inform about the educational background either, even though most of them point to a basic mastery of reading and writing competences (Fernández-Solano et al., 2019; Hatfield et al., 2017; Lindstrom et al., 2013; Smith et al., 2014), basic digital competences (Hatfield et al., 2017) or having successfully completed secondary education (Hayes et al., 2015; Oswald et al., 2017) and even post-compulsory and higher education (Mawhood et al., 1999). Nine reports were conducted in the United Sates, two of them in Spain, two in Australia and one in Japan. Only one of the reports provides evidence of previous work experience of participants (Ipsen et al., 2019). Two reports include other participants in the study, through the views of families (Hatfield et al., 2017; Powers et al., 2001) and two others take into account the views of teachers (Murray et al., 2013; Powers et al., 2001).

In terms of methodology, all research reports used a quasi-experimental design and the allocation of people to the experimental or control group was random except in three cases, two of them with non-random allocation (Lindstrom et al., 2013; Verdugo et al., 1998) and another one that does not specify this information (Mawhood \& Howlin, 1999). Regarding the activity of the control group during the implementation of the program, most research proceed with the ordinary treatment of the group. Finally, several tools were used to assess the progress of participants, and the skills most evaluated were those related to emotional wellbeing, social skills, job expectations, skills for a job interview and career planning.

Regarding the features of the intervention programs, the length varies from 10 minutes per session with a total of five sessions (Kumakazi et al., 2017), to programs cuya lasting up to 24 months (Mawhood \& Howlin, 1999). Most programs were applied individually or in small groups; while two of them combined individual and group treatment (Powers et al., 
2001; Verdugo et al., 1998). Five of the reports included the use of technologies, with online programs (Hatfield et al., 2017), mobile phones (Hayes et al., 2015; Strickland et al., 2013) or virtual reality (Kumakazi et al., 2017; Smith et al., 2014). Trainers were in most cases researchers supported by special education teachers, professional career guidance staff or human resource managers. Six of the reports indicate that trainers received specific training for the program (Lindstrom et al., 2013; Murray et al., 2013; O'Mally \& Antonelli, 2016; Oswald et al., 2017; Powers et al., 2001; Smith et al., 2014) and three of the research teams included school teachers among them (Hatfield et al., 2017; Murray et al., 2013; Verdugo et al., 1998). All reports indicate a theoretical and practical approach except the one conducted by Mawhood and Howlin (1999) where only a practical approach is considered. 
Table 1: Characteristics of the Studies Included in the Meta-Analysis - 1st Part

\begin{tabular}{|c|c|c|c|c|c|c|c|c|}
\hline \multirow[b]{2}{*}{$\begin{array}{l}\text { Authors } \\
\text { (year) }\end{array}$} & \multicolumn{5}{|c|}{ Participants } & \multicolumn{3}{|r|}{ Methodology } \\
\hline & $\begin{array}{l}\text { Age, gender } \\
\text { and country }\end{array}$ & $\begin{array}{c}\text { Type of } \\
\text { disability and IQ }\end{array}$ & $\begin{array}{l}\text { Educational } \\
\text { background }\end{array}$ & $\begin{array}{c}\text { Professional } \\
\text { training and } \\
\text { previous } \\
\text { labour } \\
\text { experience }\end{array}$ & $\begin{array}{l}\text { Other } \\
\text { recipients }\end{array}$ & $\begin{array}{c}\mathrm{N} \text { experimental, } \\
\mathrm{N} \text { control and } \\
\text { groups } \\
\text { formation } \\
\text { (random or not) }\end{array}$ & $\begin{array}{l}\text { Activity in the } \\
\text { control group }\end{array}$ & $\begin{array}{l}\text { Instrument' name, type (self-report, interview, etc.) and } \\
\text { specifically for people with disabilities }\end{array}$ \\
\hline $\begin{array}{l}\text { Fernández- } \\
\text { Solano et } \\
\text { al. (2019) }\end{array}$ & $\begin{array}{c}18-36 \\
\text { Girls }=14 \\
\text { Boys }=11 \\
\text { Spain }\end{array}$ & $\begin{array}{l}\text { Intellectual disa- } \\
\text { bility from mild } \\
\text { to moderate }\end{array}$ & $\begin{array}{l}\text { Basic skills } \\
\text { such as } \\
\text { writing, } \\
\text { reading or } \\
\text { calculation }\end{array}$ & $\begin{array}{c}\text { They were } \\
\text { enrolled in a } \\
\text { job placement } \\
\text { course }\end{array}$ & - & $\begin{array}{c}\mathrm{E}=12 \\
\mathrm{C}=13 \\
\text { Random }\end{array}$ & $\begin{array}{l}\text { Attended to } \\
\text { lessons about } \\
\text { vocational } \\
\text { guidance }\end{array}$ & $\begin{array}{l}\text { SF-36 Health Survey (Alonso et al., 1995). } \\
\text { - Self-report. No Specific. } \\
\text { Role Checklist (Colón \& Haertlein, 2002). } \\
\text { - Self-report. No Specific. } \\
\text { Participants' personal journals. } \\
\text { Focus groups. }\end{array}$ \\
\hline $\begin{array}{l}\text { Hatfield et } \\
\text { al. (2017) }\end{array}$ & $\begin{array}{c}\text { 12-18 } \\
\text { Girls }=19 \\
\text { Boys }=58 \\
\text { Australia }\end{array}$ & $\begin{array}{c}\text { Autism } \\
\text { Spectrum Disor- } \\
\text { der. Not intellec- } \\
\text { tual disability }\end{array}$ & $\begin{array}{c}\text { Basic } \\
\text { computer } \\
\text { skills and the } \\
\text { ability to wri- } \\
\text { te at a year } 5 \\
\text { reading level }\end{array}$ & - & Parents & $\begin{array}{c}\mathrm{E}=39 \\
\mathrm{C}=38 \\
\text { A quasi- } \\
\text { randomized } \\
\text { controlled trial }\end{array}$ & $\begin{array}{l}\text { Regular transition } \\
\text { planning practice }\end{array}$ & $\begin{array}{l}\text { AIR Self-Determination Scale (Wolman et al., 1994) } \\
\text { - Self-report. No Specific. } \\
\text { Career Development Inventory-Australia-Short Form } \\
\text { (Thompson et al., 1981). } \\
\text { - Self-report. No Specific. } \\
\text { Personal Wellbeing Index (PWI-SC) } \\
\text { (Cummins \& Lau, 2005). } \\
\text { - Self-report. No Specific. } \\
\text { Learning Climate Questionnaire } \\
\text { (LCQ, Williams \&Deci, 1996). } \\
\text { - Self-report. No Specific. } \\
\text { Transition Planning Objectives Scale } \\
\text { - Self-report. No Specific. }\end{array}$ \\
\hline
\end{tabular}




\begin{tabular}{|c|c|c|c|c|c|c|c|c|}
\hline $\begin{array}{l}\text { Ipsen et al. } \\
\text { (2019) }\end{array}$ & $\begin{array}{c}\text { 14-16 Girls= } \\
487 \\
\text { Boys }=941 \\
\text { USA }\end{array}$ & $\begin{array}{c}\text { Learning } \\
\text { disabilities and } \\
\text { speech and } \\
\text { language delays; } \\
18.3 \%), \\
\text { personality and } \\
\text { impulse control } \\
\text { disorders } \\
(13.8 \%), \text { autistic } \\
\text { disorders } \\
(13.9 \%), \\
\text { intellectual } \\
\text { disabilities } \\
(8.2 \%), \text { and } \\
\text { mood disorders } \\
(5.9 \%) . \\
- \\
\end{array}$ & - & $\begin{array}{l}\text { Labour } \\
\text { experience is } \\
\text { assessed in } \\
\text { both groups }\end{array}$ & - & $\begin{array}{c}\mathrm{E}=724 \\
\mathrm{C}=704 \\
\text { Random }\end{array}$ & $\begin{array}{l}\text { They receive the } \\
\text { current services } \\
\text { offered by their } \\
\text { respective state } \\
\text { transition and } \\
\text { education systems }\end{array}$ & $\begin{array}{l}\text { American Institutes for Research Self-Determination Scale } \\
\text { (AIR-SDS) (Wolman et al., 1994). } \\
\text {-Self-report. No Specific. } \\
\text { Perceived Expectations } \\
\text { Employment items } \\
\text {-Self-report. No Specific. } \\
\text { Adaptation from a single item on the Youth Risk Factor } \\
\text { Surveillance System (YBFSS) (Centers for Disease Control } \\
\text { and Prevention, 2018) } \\
\text {-Self-report. No Specific. }\end{array}$ \\
\hline $\begin{array}{l}\text { Kumakazi } \\
\text { et al. (2017) }\end{array}$ & $\begin{array}{c}18-25 \\
\text { Girls= } 3 \\
\text { Boys }=12 \\
\text { Japan }\end{array}$ & $\begin{array}{c}\text { Autism } \\
\text { spectrum } \\
\text { disorder } \\
\text { Social anxiety } \\
\text { No differences in } \\
\text { IQ between both } \\
\text { groups }\end{array}$ & - & - & - & $\begin{array}{c}\mathrm{E}=7 \\
\mathrm{C}=8 \\
\text { Random }\end{array}$ & $\begin{array}{c}\text { Read and answer } \\
\text { materials about } \\
\text { question } \\
\text { collection for } \\
\text { which was often } \\
\text { asked in real job } \\
\text { interview daily } \\
\text { for a minimum of } \\
10 \text { min }\end{array}$ & $\begin{array}{l}\text { Autism Spectrum Quotient-Japanese version (AQ-J) } \\
\text { (Wakabayashi et al., 2004). } \\
\text { - Self-report. Specific. } \\
\text { Wechsler Intelligence Scale for Children-Fourth Edition or } \\
\text { the Wechsler Adult Intelligence Scale-Third Edition. } \\
\text { - Self-report. No Specific. } \\
\text { Questionnaires scored on Likert rating scales of self-confi- } \\
\text { dence in performance } \\
\text { - Self-report. No Specific. } \\
\text { Salivary cortisol after the human interviewer sessions and } \\
\text { at the same time each day } \\
\text { - Participants provided salivary. No Specific. }\end{array}$ \\
\hline
\end{tabular}




\begin{tabular}{|c|c|c|c|c|c|c|c|c|}
\hline $\begin{array}{l}\text { Lindstrom } \\
\text { et al. (2013) }\end{array}$ & $\begin{array}{c}14-21 \\
\text { Girls }=110 \\
\text { Boys }=0 \\
\text { USA }\end{array}$ & $\begin{array}{c}\text { Disabilities and } \\
\text { other barriers- } \\
\text { Learning } \\
\text { disability (74\%) } \\
\text { Autism } \\
\text { spectrum } \\
\text { disorder (8\%) } \\
\text { Multiple } \\
\text { disabilities (7\%) } \\
\text { Intellectual } \\
\text { disability (5\%) } \\
\text { Other health } \\
\text { impairment (2\%) } \\
\text { Visual } \\
\text { impairment (2\%) } \\
\text { Hearing } \\
\text { impairment (1\%) } \\
\text { Orthopedic im- } \\
\text { pairment (1\%). }\end{array}$ & $\begin{array}{l}\text { Enrolled in } \\
\text { high school }\end{array}$ & - & & $\begin{array}{c}E=68 \\
C=42 \\
\text { Not random }\end{array}$ & $\begin{array}{l}\text { Control group } \\
\text { received the } \\
\text { typical transition } \\
\text { services that were } \\
\text { available in their } \\
\text { high schools }\end{array}$ & $\begin{array}{l}\text { PATHS survey } \\
\text { - Self-report. No Specific. } \\
\text { Two subscales from the Arc's Self-Determination Scale } \\
\text { (Wehmeyer \& Kelchner, 1995) } \\
\text { - Self-report. No Specific. } \\
\text { Subscales from the Student Engagement Inventory } \\
\text { (Appleton et al., 2006) } \\
\text { - Self-report. No Specific. } \\
\text { Teacher Support Scale (Metheny et al., 2008) } \\
\text { Vocational Skills Self- } \\
\text { Efficacy Scale (VSSE; McWhirter et al., 2000) } \\
\text { - Self-report. No Specific. } \\
\text { The Career Outcome Expectancy Scale } \\
\text { (COE; McWhirter et al. 2000). } \\
\text { - Self-report. No Specific. } \\
\text { Self-Advocacy subscale from the College Students with } \\
\text { Disabilities Campus Climate survey (Lombardi et al., 2011) } \\
\text { - Self-report. Specific. Seven focus groups } \\
\end{array}$ \\
\hline $\begin{array}{l}\text { Mawhood } \\
\text { et al. (1999) }\end{array}$ & $\begin{array}{c}18-55 \\
\text { Girls }=3 \\
\text { Boys }=47 \\
\text { United } \\
\text { Kingdom }\end{array}$ & $\begin{array}{c}\text { High } \\
\text { functioning } \\
\text { Autism or } \\
\text { Asperger Spect- } \\
\text { rum Disorder. } \\
\text { IQ } 70 \text { or above }\end{array}$ & $\begin{array}{l}\text { Range from } \\
\text { bachelor's } \\
\text { degree or } \\
\text { higher at } \\
\text { university to } \\
\text { special needs } \\
\text { courses }\end{array}$ & - & $\begin{array}{c}\text { Employers } \\
\text { Support } \\
\text { workers }\end{array}$ & $\begin{array}{l}E=30 \\
C=20\end{array}$ & - & $\begin{array}{l}\text { Psychometric battery assessing: Cognitive and linguistic } \\
\text { skills, academic attainments and social understanding. } \\
\text { - Self-report. No Specific. } \\
\text { Rosenberg Self-Esteem Inventory (Rosenberg, 1965) } \\
\text { - Self-report. No Specific. } \\
\text { A shorter battery (WAIS-R, British Picture Vocabulary } \\
\text { Scales and Expressive One-Word Picture Vocabulary Test) } \\
\text { - Self-report. No Specific. }\end{array}$ \\
\hline
\end{tabular}




\begin{tabular}{|c|c|c|c|c|c|c|c|c|}
\hline $\begin{array}{l}\text { Murray et } \\
\text { al. (2013) }\end{array}$ & $\begin{array}{c}14-18 \\
\text { Girls }=87 \\
\text { Boys }=135 \\
\text { USA }\end{array}$ & Disability & - & - & Teachers & $\begin{array}{c}E=122 \\
C=100 \\
\text { Random }\end{array}$ & $\begin{array}{c}\text { Received } \\
\text { "business-as- } \\
\text { usual" within } \\
\text { special education } \\
\text { settings }\end{array}$ & $\begin{array}{l}\text { Student report } \\
\text { Vocational outcome expectations (VOCs) (McWhirter et } \\
\text { al., 2000) } \\
\text { - Self-report. No Specific. } \\
\text { Social Skills Rating System (SSRS) (Gresham \& Elliott, } \\
\text { 1990) } \\
\text { - Self-report. No Specific. } \\
\quad \text { Teacher report } \\
\text { Occupational Skills } \\
\text { subscale on the Adaptive Behavior Inventory (Brown \& } \\
\text { Leigh, 1986). } \\
\text { Social Skills Rating System (Gresham \& Elliott, 1990). } \\
\text { Absences. } \\
\text { Teacher-rated school engagement, Research Assessment } \\
\text { Package for Schools (RAPS). } \\
\text { Teacher coverage of the WAGES curriculum }\end{array}$ \\
\hline $\begin{array}{l}\text { O'Mally et } \\
\text { al. (2016) }\end{array}$ & $\begin{array}{c}20-35 \\
\text { Girls }=32 \\
\text { Boys }=19 \\
\text { USA }\end{array}$ & $\begin{array}{c}\text { Visual } \\
\text { impairments }\end{array}$ & $\begin{array}{c}\text { Participant } \\
\text { should be } \\
\text { within a year } \\
\text { of graduation } \\
\text { and seeking } \\
\text { post-graduati- } \\
\text { on employ- } \\
\text { ment. }\end{array}$ & - & - & $\begin{array}{c}\mathrm{E}=26 \\
\mathrm{C}=25 \\
\text { Random }\end{array}$ & - & $\begin{array}{l}\text { Job-Seeking Self Efficacy (JSSE) adapted from the JSSE } \\
\text { scale for people with physical disabilities (Barlow et al., } \\
\text { 2002). } \\
\text { - Self-report. Specific. } \\
\text { Assertive Job-Hunting Survey (AJHS) (Becker, 1980). } \\
\text { - Self-report. No Specific. } \\
\text { Career Adaptability Scale (CAS) adapted from the Career } \\
\text { Futures Inventory (Rottinghaus et al., 2005) } \\
\text { - Self-report. No Specific. }\end{array}$ \\
\hline
\end{tabular}




\begin{tabular}{|c|c|c|c|c|c|c|c|c|}
\hline $\begin{array}{l}\text { Oswald et } \\
\text { al. (2017) }\end{array}$ & $\begin{array}{c}18-38 \\
\text { Girls }=13 \\
\text { Boys }=31 \\
\text { USA }\end{array}$ & $\begin{array}{c}\text { Autism } \\
\text { spectrum } \\
\text { disorder. } \\
\text { Verbal IQ } \geq 70\end{array}$ & $\begin{array}{c}\text { Completed } \\
\text { high school, } \\
\text { had spoken } \\
\text { language and } \\
\text { not exhibited } \\
\text { evidence of } \\
\text { psychosis or } \\
\text { behavioral/ } \\
\text { conduct } \\
\text { problems }\end{array}$ & - & $\begin{array}{l}\text { Each } \\
\text { partici-pant } \\
\text { selected a } \\
\text { parent or } \\
\text { close relative, } \\
\text { referred to } \\
\text { as "Social } \\
\text { Coach", to } \\
\text { participate in } \\
\text { the concur- } \\
\text { rent caregiver } \\
\text { coaching } \\
\text { group }\end{array}$ & $\begin{array}{c}E=28 \\
C=16 \\
\text { Random }\end{array}$ & $\begin{array}{l}\text { Control group } \\
\text { received the } \\
\text { ACCESS Program } \\
\text { intervention } \\
3 \text { months after } \\
\text { the two treatment } \\
\text { groups had } \\
\text { completed the } \\
\text { program }\end{array}$ & $\begin{array}{l}\text { Informant-Report Measures Adaptive Behavior Assessment } \\
\text { System-Adult Form, Third Edition (ABAS-3) (Harrison \& } \\
\text { Oakland, 2015) } \\
\text { - Self-report by Social coach. No Specific. } \\
\text { Seven Component Self-Determination Skills Survey (Carter } \\
\text { et al., 2013) } \\
\text { - Self-report by Social coach. No Specific. } \\
\text { Self-Report Measures } \\
\text { Coping Self-Efficacy Scale (CSES) (Chesney et al., 2006) } \\
\text { - Self-report. No Specific. } \\
\text { ASEBA Adult Self-Report (ASR) (Achenbach \& Rescorla, } \\
\text { 2003) } \\
\text { - Self-report. No Specific }\end{array}$ \\
\hline $\begin{array}{l}\text { Powers et } \\
\text { al. (2001) }\end{array}$ & $\begin{array}{c}14-17 \\
\text { Girls }=13 \\
\text { Boys }=30 \\
\text { USA }\end{array}$ & $\begin{array}{c}\text { Diverse } \\
\text { disabilities }\end{array}$ & - & $\begin{array}{l}\text { With regard } \\
\text { to attendance } \\
\text { at educational } \\
\text { or transition } \\
\text { planning } \\
\text { meetings, } \\
43 \% \text { of the } \\
\text { treatment } \\
\text { group had } \\
\text { attended their } \\
\text { prior year's } \\
\text { meeting while } \\
41 \% \text { of the } \\
\text { wait list group } \\
\text { had attended } \\
\text { their meeting. }\end{array}$ & - & $\begin{array}{c}E=21 \\
\mathrm{C}=22 \\
\text { Random }\end{array}$ & $\begin{array}{l}\text { Control group } \\
\text { would participate } \\
\text { during the } \\
\text { following } \\
\text { semester }\end{array}$ & $\begin{array}{l}\text { Educational Planning Assessment (EPA) (developed by this } \\
\text { research) } \\
\text { - Self-report. No Specific. } \\
\text { The Transition Awareness Survey (Martin \& Marshall, } \\
\text { 1993) } \\
\text { - Self-report. No Specific. } \\
\text { The Family Empowerment Scale (Koren et al., 1992) } \\
\text { - Self-report. Specific. } \\
\text { Observational coding system evaluated student } \\
\text { participation in transition planning meetings. }\end{array}$ \\
\hline
\end{tabular}




\begin{tabular}{|c|c|c|c|c|c|c|c|c|}
\hline $\begin{array}{l}\text { Smith et al. } \\
\text { (2014) }\end{array}$ & $\begin{array}{c}18-31 \\
\text { Girls= } 5 \\
\text { Boys= } 21 \\
\text { USA }\end{array}$ & $\begin{array}{l}\text { Autism } \\
\text { spectrum } \\
\text { disorder }\end{array}$ & $\begin{array}{c}\text { (1) have at } \\
\text { least a 6th } \\
\text { grade reading } \\
\text { level }\end{array}$ & - & - & $\begin{array}{c}\mathrm{E}=16 \\
\mathrm{C}=10 \\
\text { Random }\end{array}$ & $\begin{array}{c}\text { Treatment as } \\
\text { usual }\end{array}$ & $\begin{array}{l}\text { Repeatable battery for the assessment of } \\
\text { neuropsychological } \\
\text { status (RBANS) (Randolph et al., 1998) } \\
\text { Bell-Lysaker emotion recognition task (BLERT) (Bell et } \\
\text { al., 1997) }\end{array}$ \\
\hline $\begin{array}{l}\text { Strickland } \\
\text { et al. (2013) }\end{array}$ & $\begin{array}{c}16-19 \\
\text { Girls }=0 \\
\text { Boys }=22 \\
\text { USA }\end{array}$ & $\begin{array}{l}\text { High functio- } \\
\text { ning Autism } \\
\text { Spectrum } \\
\text { Disorders and } \\
\text { Asperger's } \\
\text { Disorder. } \\
\text { Not intellectual } \\
\text { disability }\end{array}$ & & - & - & $\begin{array}{c}\mathrm{E}=11 \\
\mathrm{C}=11 \\
\text { Random }\end{array}$ & None & $\begin{array}{l}\text { Interview skills rating instruments } \\
\text { - A human resources professional completed the rating } \\
\text { scales based on participant. No Specific. } \\
\text { Social responsiveness scale (Constantino \& Gruber, 2005). } \\
\text { - A human resource professional completed the rating } \\
\text { scales based on participant performance. } \\
\text { - It allows the quantification of symptomatology associated } \\
\text { with ASD. }\end{array}$ \\
\hline $\begin{array}{l}\text { Verdugo et } \\
\text { al. (1998) }\end{array}$ & $\begin{array}{c}18-45 \\
\text { Girls }=35 \\
\text { Boys }=49 \\
\text { Spain }\end{array}$ & $\begin{array}{c}\text { Physical } \\
\text { disabilities and } \\
\text { deafness }\end{array}$ & $\begin{array}{l}\text { They are } \\
\text { studying in a } \\
\text { professional } \\
\text { rehabilitation } \\
\text { center }\end{array}$ & $\begin{array}{l}\text { Students of } \\
\text { fields related } \\
\text { to office } \\
\text { work, artistic } \\
\text { fields and } \\
\text { the practical } \\
\text { activities }\end{array}$ & - & $\begin{array}{c}E=56 \\
C=28 \\
\text { Not random }\end{array}$ & - & $\begin{array}{l}\text { My Vocational } \\
\text { Situation, (Holland et al., 1980). } \\
\text { - Self-report. No Specific. } \\
\text { The Employability Maturity Interview, EMI; (Morelock et } \\
\text { al., 1987) } \\
\text { - Self-report. No Specific. } \\
\text { Vocational Self-Awareness, (Farley, 1988) } \\
\text { - Self-report. No Specific. } \\
\text { Self-Image questionnaire Revised, OSIQR, (Offer et al., } \\
\text { 1992). } \\
\text { - Self-report. No Specific. }\end{array}$ \\
\hline
\end{tabular}




\begin{tabular}{|c|c|c|c|c|c|c|c|c|}
\hline $\begin{array}{l}\text { Hayes et al. } \\
(2015)\end{array}$ & $\begin{array}{c}17-18 \\
\text { Girls }=2 \\
\text { Boys }=13 \\
\text { USA }\end{array}$ & $\begin{array}{l}\text { Autism } \\
\text { Spectrum } \\
\text { Disorders }\end{array}$ & $\begin{array}{l}\text { Senior year of } \\
\text { high school or } \\
\text { had graduated } \\
\text { high school }\end{array}$ & $\begin{array}{l}\text { Receiving } \\
\text { services } \\
\text { from the } \\
\text { transition } \\
\text { program }\end{array}$ & - & $\begin{array}{c}\mathrm{E}=8 \\
\mathrm{C}=7 \\
\text { Random }\end{array}$ & None & $\begin{array}{l}\text { A rubric } \\
\text { Researchers transcribed } \\
\text { the research interviews for qualitative analysis }\end{array}$ \\
\hline
\end{tabular}

Note. IQ= Intelligence Quotient

Table 1: Characteristics of the Studies Included in the Meta-Analysis - 2nd Part

\begin{tabular}{|c|c|c|c|c|c|c|c|c|c|}
\hline \multirow{3}{*}{$\begin{array}{c}\text { Authors } \\
\text { (year) }\end{array}$} & \multicolumn{9}{|c|}{ Intervention } \\
\hline & \multirow{2}{*}{$\begin{array}{l}\text { Name of the } \\
\text { program or } \\
\text { intervention }\end{array}$} & \multirow{2}{*}{$\begin{array}{c}\text { Duration } \\
\text { Frequency and total }\end{array}$} & \multirow{2}{*}{$\begin{array}{c}\text { Proportion } \\
\begin{array}{c}\mathrm{N} / \text { group } \\
\text { intervention }\end{array}\end{array}$} & \multirow{2}{*}{$\begin{array}{c}\begin{array}{c}\text { Use of } \\
\text { technology }\end{array} \\
\text { Used or not } \\
\text { Type } \\
\text { Access }\end{array}$} & \multicolumn{3}{|c|}{ Teaching profile } & \multirow{2}{*}{$\begin{array}{c}\text { Procedure } \\
\text { Theory } \\
\text { and/or } \\
\text { Practice }\end{array}$} & \multirow{2}{*}{$\begin{array}{c}\text { Assessmen } \\
\begin{array}{c}\text { Process or } \\
\text { final }\end{array}\end{array}$} \\
\hline & & & & & $\begin{array}{l}\text { Background } \\
\text { education }\end{array}$ & $\begin{array}{l}\text { Knowledge in } \\
\text { research topic }\end{array}$ & $\begin{array}{l}\text { Relationship } \\
\text { with } \\
\text { participants }\end{array}$ & & \\
\hline $\begin{array}{l}\text { Fernández- } \\
\text { Solano et } \\
\text { al. (2019) }\end{array}$ & $\begin{array}{l}\text { Occupational Self-Analysis } \\
\text { Programme. } \\
\text { An adaptation of the 'Age } \\
\text { Doing' programme (Ro- } \\
\text { dríguez-Bailón et al. 2016) }\end{array}$ & $\begin{array}{l}90 \text { minutes/week } \\
9 \text { months }\end{array}$ & Group & Not used & - & - & - & $\begin{array}{l}\text { Theory } \\
\text { and/or } \\
\text { Practice }\end{array}$ & $\begin{array}{l}\text { Initial and } \\
\text { final }\end{array}$ \\
\hline $\begin{array}{l}\text { Hatfield et } \\
\text { al. (2017) }\end{array}$ & $\begin{array}{l}\text { Better OutcOmes \& Successful } \\
\text { Transitions for Autism } \\
\left(\text { BOOST- } A^{m *}\right)\end{array}$ & 12 months & Individually & $\begin{array}{c}\text { Used } \\
\text { Online } \\
\text { program } \\
\text { Closed } \\
\text { Individual } \\
\text { login }\end{array}$ & - & - & School staff & $\begin{array}{l}\text { Theory } \\
\text { and } \\
\text { practice }\end{array}$ & $\begin{array}{l}\text { Initial and } \\
\text { final }\end{array}$ \\
\hline
\end{tabular}




\begin{tabular}{|c|c|c|c|c|c|c|c|c|c|}
\hline $\begin{array}{l}\text { Ipsen et al. } \\
(2019)\end{array}$ & $\begin{array}{l}\text { Achieving Success by } \\
\text { Promoting Readiness for } \\
\text { Education and Employment } \\
\text { (ASPIRE) consortium, one of } \\
\text { the six PROMISE sites. } \\
\end{array}$ & $\begin{array}{l}- \\
12 \text { months } \\
24 \text { months }\end{array}$ & Individually & Not used & $\begin{array}{l}\text { Vocational } \\
\text { Rehabilitation } \\
\text { counselor }\end{array}$ & - & - & $\begin{array}{l}\text { Theory } \\
\text { and } \\
\text { practice }\end{array}$ & $\begin{array}{c}\text { Initial, } 12 \\
\text { months } \\
\text { later and } \\
24 \text { months } \\
\text { later } \\
\end{array}$ \\
\hline $\begin{array}{l}\text { Kumakazi } \\
\text { et al. (2017) }\end{array}$ & $\begin{array}{l}\text { Android Robot-Mediated } \\
\text { Mock Job } \\
\text { Interview Sessions }\end{array}$ & $\begin{array}{l}10 \text { minutes/day } \\
5 \text { days }\end{array}$ & Individually & $\begin{array}{c}\text { Used } \\
\text { Android robot }\end{array}$ & - & - & - & $\begin{array}{l}\text { Theory and } \\
\text { practice }\end{array}$ & $\begin{array}{l}\text { Initial and } \\
\text { final }\end{array}$ \\
\hline $\begin{array}{l}\text { Lindstrom } \\
\text { et al. (2013) }\end{array}$ & $\begin{array}{l}\text { Postschool Achievement } \\
\text { Through Higher Skills - PATHS }\end{array}$ & $\begin{array}{l}\text { 50-minute class } \\
77 \text { lessons } \\
\text { 18-week semester }\end{array}$ & Small groups & Not used & - & $\begin{array}{c}\text { All PATHS } \\
\text { instructors attended } \\
\text { an initial 1.5-day } \\
\text { workshop, followed } \\
\text { by three } 2 \text {-hour } \\
\text { training sessions } \\
\text { throughout the } \\
\text { 18-week } \\
\text { implementation } \\
\text { period }\end{array}$ & $\begin{array}{l}\text { No } \\
\text { relationship }\end{array}$ & $\begin{array}{l}\text { Theory and } \\
\text { practice }\end{array}$ & $\begin{array}{l}\text { Initial and } \\
\text { final }\end{array}$ \\
\hline $\begin{array}{l}\text { Mawhood } \\
\text { et al. (1999) }\end{array}$ & $\begin{array}{l}\text { Supported employment project } \\
\text { for high-functioning adults } \\
\text { with autism }\end{array}$ & $\begin{array}{l}\text { - } \\
\text { Range from } 5 \text { to } 24 \\
\text { months }\end{array}$ & $\begin{array}{l}\text { Individually in } \\
\text { a company }\end{array}$ & Not used & - & - & $\begin{array}{l}\text { Support } \\
\text { worker }\end{array}$ & Practice & $\begin{array}{l}\text { Initial and } \\
\text { final }\end{array}$ \\
\hline $\begin{array}{l}\text { Murray et } \\
\text { al. (2013) }\end{array}$ & $\begin{array}{l}\text { Working at Gaining } \\
\text { Employment Skills (WAGES) }\end{array}$ & $\begin{array}{c}3 \text { and } 4 \text { days per week } \\
\text { Approx. } \\
4.5 \text { months } \\
\text { (January-May) }\end{array}$ & $\begin{array}{l}\text { Activities that } \\
\text { encourage } \\
\text { students to } \\
\text { participate } \\
\text { cooperatively }\end{array}$ & Not used & $\begin{array}{l}\text { Special education } \\
\text { teachers, } \\
\text { vocational } \\
\text { rehabilitation } \\
\text { counsellors, or } \\
\text { other school } \\
\text { personnel }\end{array}$ & $\begin{array}{l}\text { All teachers were } \\
\text { provided a free copy } \\
\text { of the WAGES } \\
\text { curriculum and } \\
\text { participated in a } \\
\text { paid (US } \$ 35 \text { per } \\
\text { hour) 2-day training } \\
\text { about WAGES }\end{array}$ & $\begin{array}{l}\text { Their } \\
\text { teachers }\end{array}$ & $\begin{array}{l}\text { Theory and } \\
\text { practice }\end{array}$ & $\begin{array}{l}\text { Initial and } \\
\text { final }\end{array}$ \\
\hline
\end{tabular}




\begin{tabular}{|c|c|c|c|c|c|c|c|c|c|}
\hline $\begin{array}{l}\text { O'Mally et } \\
\text { al. (2016) }\end{array}$ & $\begin{array}{l}\text { Career Mentoring on } \\
\text { Employment Outcomes }\end{array}$ & $\begin{array}{c}- \\
1 \text { year }\end{array}$ & - & Not used & $\begin{array}{c}\text { Mentors } \\
\text { included those } \\
\text { who were } \\
\text { currently employed } \\
\text { or recently retired, } \\
\text { legally blind, and } \\
\text { living in the United } \\
\text { States }\end{array}$ & $\begin{array}{c}\text { Employment } \\
\text { mentoring manual } \\
\text { A 20-page } \\
\text { manual in electronic } \\
\text { format to } \\
\text { students and their } \\
\text { mentors }\end{array}$ & - & $\begin{array}{l}\text { Theory } \\
\text { and } \\
\text { practice }\end{array}$ & $\begin{array}{l}\text { Initial and } \\
\text { final }\end{array}$ \\
\hline $\begin{array}{l}\text { Oswald et } \\
\text { al. (2017) }\end{array}$ & $\begin{array}{l}\text { Acquiring Career, Coping, } \\
\text { Executive control, Social Skills } \\
\text { (ACCESS) Program }\end{array}$ & $\begin{array}{c}1 \text { hour and a half } 19 \\
\text { sessions }\end{array}$ & Small groups & Not used & $\begin{array}{l}\text { The supervisor was } \\
\text { a licensed } \\
\text { psychologist.The } \\
\text { Participant Group } \\
\text { leader, the Social } \\
\text { Coach Group } \\
\text { leader and the } \\
\text { co-facilitators have } \\
\text { advanced studies } \\
\text { and prior } \\
\text { experience } \\
\text { working with these } \\
\text { people. } \\
\end{array}$ & Yes & - & $\begin{array}{l}\text { Theory } \\
\text { and/or } \\
\text { Practice }\end{array}$ & $\begin{array}{l}\text { Initial and } \\
\text { final }\end{array}$ \\
\hline $\begin{array}{l}\text { Powers et } \\
\text { al. (2001) }\end{array}$ & TAKE CHARGE For the Future & $\begin{array}{l}\text { The intervention (a) } \\
\text { individual, } 50 \\
\text { minute bi-weekly } \\
\text { coaching sessions for } \\
\text { youths, (b) monthly } \\
\text { community-based work- } \\
\text { shops, (c) community } \\
\text { activities, (d) telephone } \\
\text { and home visit support } \\
\text { for parents, and (e) } \\
\text { in-service education for } \\
\text { transition staff } 4 \text { months }\end{array}$ & $\begin{array}{l}\text { Individually } \\
\text { and small } \\
\text { groups }\end{array}$ & Not used & $\begin{array}{l}\text { The team included } \\
\text { an experienced } \\
\text { secondary } \\
\text { educator, a parent } \\
\text { support staff } \\
\text { person, and a peer } \\
\text { counselor from } \\
\text { the community } \\
\text { independent living } \\
\text { program. }\end{array}$ & $\begin{array}{l}\text { The research teams } \\
\text { participated in a } \\
\text { three-day } \\
\text { training } \\
\text { program }\end{array}$ & $\begin{array}{l}\text { Research } \\
\text { teams }\end{array}$ & $\begin{array}{l}\text { Theory } \\
\text { and/or } \\
\text { Practice }\end{array}$ & $\begin{array}{l}\text { Initial and } \\
\text { final }\end{array}$ \\
\hline
\end{tabular}




\begin{tabular}{|c|c|c|c|c|c|c|c|c|c|}
\hline $\begin{array}{l}\text { Smith et al. } \\
\text { (2014) }\end{array}$ & $\begin{array}{l}\text { Virtual reality job interview } \\
\text { training }\end{array}$ & $\begin{array}{l}5 \text { sessions of } 2 \text { hours } \\
2 \text { weeks }\end{array}$ & Individually & $\begin{array}{l}\text { Used } \\
\text { Virtual reality } \\
\text { Closed }\end{array}$ & - & $\begin{array}{l}\text { Two research staff } \\
\text { members were } \\
\text { trained to } \\
\text { administer the } \\
\text { intervention using a } \\
\text { checklist devised by } \\
\text { the scientific team }\end{array}$ & - & $\begin{array}{l}\text { Theory and } \\
\text { practice }\end{array}$ & $\begin{array}{l}\text { Initial and } \\
\text { final }\end{array}$ \\
\hline $\begin{array}{l}\text { Strickland } \\
\text { et al. (2013) }\end{array}$ & JobTIPS employment program & - & Individually & $\begin{array}{l}\text { Used Online } \\
\text { program } \\
\text { Closed }\end{array}$ & $\begin{array}{c}\text { Human resources } \\
\text { professional }\end{array}$ & - & - & $\begin{array}{l}\text { Theory and } \\
\text { practice }\end{array}$ & $\begin{array}{l}\text { Initial and } \\
\text { final }\end{array}$ \\
\hline $\begin{array}{l}\text { Verdugo et } \\
\text { al. (1998) }\end{array}$ & $\begin{array}{l}\text { Program of vocational evaluati- } \\
\text { on and guidance }\end{array}$ & $\begin{array}{c}18 \text { sessions of } 1 \text { hour and } \\
\text { a half } \\
27 \text { hours }\end{array}$ & $\begin{array}{l}\text { Individually } \\
\text { and groups }\end{array}$ & Not used & $\begin{array}{l}\text { Teachers, the } \\
\text { signed language } \\
\text { teacher and other } \\
\text { volunteers }\end{array}$ & - & $\begin{array}{c}\text { Teachers } \\
\text { from the } \\
\text { center and } \\
\text { other } \\
\text { volunteers }\end{array}$ & $\begin{array}{l}\text { Theory and } \\
\text { practice }\end{array}$ & $\begin{array}{l}\text { Initial and } \\
\text { final }\end{array}$ \\
\hline $\begin{array}{l}\text { Hayes et al. } \\
(2015)\end{array}$ & VidCoach & 1 month & Individually & $\begin{array}{l}\text { Used Mobile } \\
\text { video } \\
\text { modelling and } \\
\text { prompting } \\
\text { application } \\
\text { Closed }\end{array}$ & - & - & - & $\begin{array}{l}\text { Theory } \\
\text { and } \\
\text { practice }\end{array}$ & $\begin{array}{l}\text { Initial and } \\
\text { final }\end{array}$ \\
\hline
\end{tabular}




\subsection{Mean Effect Size and Heterogeneity Analysis}

Table 2 provides the effect size and the statistics of heterogeneity of the meta-analysis conducted. First, we analyzed the medium effect of the group of all studies upon data provided by people with functional diversity. Second, we included two independent metaanalysis taking into consideration results provided by relatives and teachers respectively. Third, we conducted five meta-analysis upon skills most often developed in these programs and for which we had results in at least five studies. These consisted of interview, career planning, emotional welfare, social skills and job expectations.

The effectiveness of treatment was measured through the effect size. For the group of all studies, the average effect size was a value of .38, which indicates that programs that develop labor skills for people with functional diversity proved effective for the experimental group. Accordingly, results provided by relatives and teachers revealed values of global average effect size of .43 and .53 respectively in favor of the experimental group. These effect sizes proved of low magnitude for the group of all reports and the viewpoint of relatives, while the magnitude proved moderate for the case of teachers.

Regarding the areas promoted by the programs, an average effect size in favor of the experimental group was found in interview skills, career planning and job expectations. The magnitude of effect was moderate for career planning $(d=.66)$ and low for interview skills $(d=.42)$, while it did not achieve a practical relevance in terms of job expectations $(d=.09)$.

The average global effect for all studies showed a moderate magnitude of variability $\left(I^{2}=74 \%\right)$, as well as for the area of career planning $\left(I^{2}=68 \%\right)$. Furthermore, the degree of heterogeneity was also moderate when the relatives reported $\left(I^{2}=68 \%\right)$, while it was high in the case of teachers $\left(I^{2}=87 \%\right)$.

If the homogeneity test proves significative and the index $I^{2}$ indicates heterogeneity in the size effect, it is convenient to conduct analysis of possible moderating variables that explain the heterogeneity. These analyses were conducted only on the set of all studies ( $k=67$ studies), not being applied to data on the different areas nor those reported by relatives ( $k=9$ studies) nor teachers ( $k=5$ studies) due to the reduced numbers within these categories. 
Table 2: Effect Size and Analysis of Heterogeneity in the Posttest

\begin{tabular}{|c|c|c|c|c|c|c|c|c|}
\hline & \multirow[t]{2}{*}{$k$} & \multirow[t]{2}{*}{$d$} & \multicolumn{2}{|c|}{$95 \% \mathrm{CI}$} & \multirow[t]{2}{*}{$Q$} & \multirow[t]{2}{*}{$d f$} & \multirow{2}{*}{$\begin{array}{c}\text { Test for } \\
\text { overall } \\
\text { effect }(z, p)\end{array}$} & \multirow[t]{2}{*}{$I^{2}$} \\
\hline & & & $\mathrm{d}_{1}$ & $d_{u}$ & & & & \\
\hline Global effect & 67 & .38 & .28 & .48 & 258.09 & 66 & $7.45,<.001$ & $74 \%$ \\
\hline Family & 9 & .43 & .16 & .69 & 25.22 & 8 & $3.17,<.05$ & $68 \%$ \\
\hline Teachers & 5 & .53 & .14 & .92 & 38.03 & 4 & $3.88,<.05$ & $87 \%$ \\
\hline Interview skills & 13 & .42 & .10 & .75 & 19.03 & 12 & $2.53,<.05$ & $37 \%$ \\
\hline Career planning & 8 & .66 & .34 & .98 & 22.02 & 7 & $4.03,<.001$ & $68 \%$ \\
\hline Emotional welfare & 6 & .22 & -.14 & .59 & 10.24 & 5 & $1.21, .23$ & $51 \%$ \\
\hline Social skills & 6 & .30 & -.06 & .65 & 21.94 & 5 & $1.65, .10$ & $77 \%$ \\
\hline Labor expectations & 5 & .09 & .01 & .17 & 4.73 & 4 & $2.20,<.05$ & $15 \%$ \\
\hline
\end{tabular}

Note. $k=$ number of studies, $d=$ mean effect size, CI= confidence interval, $d_{1}$ and $d u=$ lower and upper confidential limits of the $95 \%$ confidence interval around the mean effect size, $Q=$ homogeneity test, $d f=$ degrees of freedom of $Q$ statistic, $I^{2}=$ heterogeneity index

\subsection{Analysis of Moderating Variables}

The analysis of moderating variables was applied to the set of studies with data reported by people with functional diversity. Thirteen variables were tested, which are classified in participants' variables (age of participants, type of disability, educational background, other participants in the study, training of the instructors, relation of the participants with the instructors), treatment's variables (length of the program, use of technologies, type of technologies), methodological characteristics (type of allocation to control/experimental group, type of grouping for the intervention) and extrinsic characteristics (publication year, country). The inter-category homogeneity statistic was significant for the variables type of disability, educational background, length and country.

Regarding participant's variables the type of disability and educational background were significant moderating variables. With regard to the type of disability, we compared the efficacy of treatments by differentiating four groups according to the type of disability or disorder of participants in the programs: (1) Autistic spectrum disorder, (2) intellectual disability, (3) physical or sensory disability and (4) several disabilities or disorders in the same group and program. The results revealed statistically significant differences between the four groups $\left(Q_{\mathrm{b}}=10.68, p=.01\right)$, reaching a larger effect size of intervention when groups were shaped by people with intellectual disability ( $d=.84$; 95\% IC: .38 and 1.31) before the remaining types whose impact was lower, despite also significative.

Regarding educational background, we distinguished two groups (1) basic educational level or young people enrolled in secondary education and (2) intermediate educational background with a degree in secondary education or higher education studies. We obtained 
statistically significative differences between groups $\left(Q_{b}=6.07 ; p=.01\right)$, where the group with lower educational background achieved a larger effect size ( $d=.62$; 95\% IC: .36 and .88 ).

Regarding treatment's variables, the length of the programs was a significant moderating variable. We distinguished four groups (1) less than 10 sessions or shorter than a month, (2) 11 to 20 sessions or between two to five months, (3) six to twelve months and (4) beyond a year. Statistically significative differences resulted among the average effects of the groups $\left(Q_{\mathrm{b}}=7.20 ; p=.03\right)$, where programs between six and twelve months $(d=.68 ; 95 \%$ IC: .34 and 1.01) proved more effective than shorter and longer ones.

Finally, regarding the extrinsic characteristics, some words about the four countries in the studies (1) United States of America, (2) Spain, (3) Australia and (4) Japan; results revealed statistically significative differences among the groups $\left(Q_{b}=8.50 ; p=.04\right)$, where Spanish studies $(d=.58$; 95\% IC: .36 and .80$)$ and Australian ones ( $d=.55$; 95\% IC: -.08 and 1.17$)$ obtained a larger impact in comparison to the USA and Japan.

Table 3: Moderating Variables

\begin{tabular}{|c|c|c|c|c|c|c|}
\hline \multicolumn{4}{|c|}{ Moderating variables } & \multicolumn{2}{|c|}{ IC 95\% } & \multirow[t]{2}{*}{ Results } \\
\hline & & $k$ & $d$ & $d_{l}$ & $d_{u}$ & \\
\hline \multirow{4}{*}{$\begin{array}{c}\text { Type of } \\
\text { disability }\end{array}$} & ASD & 28 & .35 & .09 & .61 & $Q_{b}=10.68, p=.01$ \\
\hline & Intellectual disability & 10 & .84 & .38 & 1.31 & $I^{2}=71.9 \%$ \\
\hline & Physical o sensory disability & 12 & .45 & .28 & .61 & \\
\hline & Several & 17 & .21 & .10 & .32 & \\
\hline Educational & Basic or high school skills & 25 & .62 & .36 & .88 & $Q_{b}=6.07 ; p=.01$ \\
\hline background & Secondary completed or higher & 16 & .22 & .03 & .41 & $I^{2}=83.5 \%$ \\
\hline \multirow[t]{4}{*}{ Duration } & Less than 10 sessions or up to 1 month & 13 & .22 & -.04 & .47 & $Q_{b}=7.20 ; p=.03$ \\
\hline & Between $11-20$ sessions or 2-5 months & 26 & .38 & .23 & .52 & $I^{2}=72.2 \%$ \\
\hline & Between 6-12 months & 20 & .68 & .34 & 1.01 & \\
\hline & More than a year & 6 & .05 & .00 & .10 & \\
\hline \multirow[t]{4}{*}{ Country } & USA & 38 & .26 & .16 & .36 & $Q_{b}=8.50 ; p=.04$ \\
\hline & Spain & 19 & .58 & .36 & .80 & $I^{2}=64.7 \%$ \\
\hline & Australia & 7 & .55 & -.08 & 1.17 & \\
\hline & Japan & 2 & -.11 & -.83 & .62 & \\
\hline
\end{tabular}

Note. ASD $=$ Autism Spectrum Disorder, $k=$ number of studies, $d=$ mean effect size. $d_{1}$ and $d_{\mathrm{u}}=$ lower and upper confidential limits of the $95 \%$ confidence interval around the mean effect size, $Q_{b}=$ inter-category homogeneity statistic,

$I^{2}=$ heterogeneity index 


\section{Discussion}

The issue we have addressed in this paper is currently being discussed by the academy, and $85.7 \%$ of the articles reviewed have been published in the last decade. The fact that such research is addressed by the scientific community is also an indicator of the growing weight of fostering employability among people with disabilities in order to facilitate their access into the labor market, as one more dimension having an impact upon social inclusion: There is enough employability appraisal nowadays to allow for research to be conducted in the field. That being the context for our research question, we can now discuss our results.

First, we can state that programs have a positive effect upon the experimental groups as we can see in tables 2 and 3 Results obtained from the views of relatives as teachers provide a statistically significant mean effect in favor of the experimental group. Furthermore, if we take into account the skills developed in the different programs, we identified a mean effect size in favor of the experimental group in the areas of career planning, interview skills and professional expectations; while no significative effect was found in comparison with the results of the control group for dimensions such as emotional welfare or social skills. Effect sizes we found vary from small magnitudes for all of the reports, family perception and interview skills, to a moderating magnitude for the perception of teachers and career planning. We did not find any large magnitude.

Nevertheless, we must consider that the struggle for social inclusion in the field of disabilities has been a history of small yet steady advances, rather than sudden improvements nor high-impact solutions (López-Bastías, 2019). If we look back thirty years, work integration of people with disabilities was not even an expectation, and we are now discussing not just training for an occupation but even development of employability skills, the focus of our review. This is a step beyond occupational training, as employability embeds social and personal skills that are useful both in work and in everyday life domains (Llinares et al., 2020a).

Second, we have to consider the assessment of teachers and trainers, who share a higher perception of the impact of the programs under review. This is relevant for the fact that teachers and trainers are able to compare and contrast among people who have taken part in the experimental programs and those who have not, but also for they are able to compare among these people ready to improve their employability and others with similar handicaps whose employability is lower and therefore not appropriate to consider further preparation for integration in the labor market. Given that employability can be measured (most employability assessment tools synthetize achievement in terms of grades), the perception and evaluation of teachers becomes even more relevant; as families and people with disabilities themselves do not have that comparative context. Teachers have specific training, they are aware of labor market conditions, they know the results of integration into the labor market of former cohorts of people attending their programs, and they can assess people's employability with less personal engagement and larger group reference. 
If we move now in the specific detail regarding dimensions, our results do not match the previous study upon social skills (Park et al., 2016), as we have not found significative impact in this domain. We suggest different explanations for this result: First, our study has focused not only upon this dimension, and once we have conducted a broader search, we may have missed some study. This is however a common limitation in meta-analysis studies known as selection and publication bias (Sandoya, 2008) despite our methodological approach is robust enough, as we have detailed above. However, social skills are not only trained for employability purposes, and they can also be part of other integration and training programs (Bundock \& Hewitt, 2017).

Furthermore, social skills have the opportunity to be developed in most everyday activities also out of training programs, this being an issue that cannot be measured under experimental conditions: The more participation a person has in ordinary life in society, the more chances to develop and improve social skills. In fact, there is literature on employability that considers the advantages of developing social skills out of specific training measures (Ibáñez \& Mudarra, 2005).

Anyhow, the effect size has resulted significatively positive in favor of the experimental group in the domains that can be linked to a greater extent to work, such as career planning, interview skills and labor expectations. This is relevant for these are issues that, in contrast to general social skills, are labor-specific and are part, as such, of any vocational education and training program also for all kinds of people as well as for all levels of qualification (Hedley et al., 2017; Jordan et al., 2016; Lee et al., 2018; Walsh et al., 2017). These are crucial in the preparation for employment and therefore these results are particularly relevant in our study, as they do not deal just with social inclusion but directly with integration into the labor market.

In summary, these results contribute to encourage the research community (and practitioners in first place) to keep working in other dimensions surrounding employability such as the emotional wellbeing and overall health, as these constitute the basis to strengthen the impact of all other variables (Cavadel et al., 2017; Llinares et al., 2020a, 2020b).

Regarding the moderating variables, we found that four of the 13 variables analyzed had a positive impact upon the effectiveness of the interventions: Where the participants have a lower educational level and intellectual disability (participants moderating variables); where the duration of the programs is between 6 and 12 months (treatment moderating variable); and those from Spain or Australia (extrinsic moderating variable). It becomes evident that participant variables, treatment variables and extrinsic characteristics do play a role, while methodological features do not.

Lower educational level has a direct impact not only upon career expectations, but also upon the qualification level to which one can prepare and, therefore, it introduces serious limitations in prospective employments which are less demanding not only in terms of occupational skills but also of employability skills. 
Intellectual disability is well studied, it is different to mental illness and subject to medical measurement that indicates degrees of impairment that have an impact upon job choices, which is sometimes twofold: They can be obstacles to find proper jobs, though there are companies that sometimes search for candidates with certain percentage of disability as this provides social security and fiscal benefits for the company.

Duration of the training programs makes a difference as the impact of short-term programs upon long-term issues like career planning, career expectations and chances to engage in the job-search process, that are not as frequent as desirable and are also subject to labor market chances, as we have stated just above.

The country effect can be weighed by two different conditions: First, the cultural consideration that functional diversity has among the population and the overall awareness of inclusion rights and practices. This will be consequently reflected in employment and employability policies. Second, the productive fabric of the different countries indicates different labor market structures as well as labor relations which are also directly related to the chances to hire people with functional diversity.

At this point, some limitations of this meta-analysis should be mentioned. As in this study, most conventional meta-analytic procedures assume independence among the effect sizes (Cheung, 2014, 2019). The homogenization of the results was carried out by applying the index of effect size typified mean change difference. Homogeneous and comparable quantitative index independently of the different tests used in the different studies, interpreting them as typical units of separation between the means of the two groups (Sánchez-Meca \& Botella, 2010). However, averaging the effect sizes or selecting one effect size per study may lead to missed opportunities to utilize all available data. In order to overcome this limitation, multivariate meta-analysis and three-level meta-analysis have been proposed as a future research line to handle non-independent effect sizes (Cheung, 2019). Another limitation was the scarce number of studies that fulfilled the selection criteria. Consequently, results need to be interpreted with caution pending the publication of new studies in this field.

Finally, we would like to focus upon the social and educational implications of the metaanalysis we have conducted. First, without doubt, more experimental research is needed in order to contrast the results showed so far. Furthermore, it would be advisable to produce research that is applied to people with similar handicaps in different countries, something which is possible particularly in short-term training programs, and several of them were mentioned in our study. Second, this is also possible in relation to certain disabilities which, in a way, contribute to homogenize the population and therefore the groups of people that attend such training interventions, allowing for more experimental design than research upon training programs in other domains where there is much wider variability of conditions that may have an impact upon the effects of vocational or employability programs. Third, longterm training programs are advisable if we want to strengthen issues like career planning 
and the development of vocational expectations and identities, as these are dimensions that demand educational rather than training interventions.

Programs that support and develop employability and that are conducted upon experimental conditions do have a positive impact upon young people with functional diversity, and this is a promising result in relation to the expansion of such programs in order to facilitate their access into the labor market and hence more and better chances for social inclusion and participation as adult citizens.

Our contribution has intended to advance knowledge in this terrain, and it has also allowed us to identify the scarcity of programs and of experimental research in an area in which, due to the medical diagnostic behind several disabilities, they might apply better than in other circumstances surrounding vulnerable transitions, such as those caused by social instead of biological factors.

\section{Acknowledgement}

The authors thank the Department of Didactics and School Organization (University of Valencia) for its support for Carolina Gonzálvez to conduct a postdoctoral research stay under the supervision of Professor Fernando Marhuenda from 27 May 2019 to 28 June 2019, when Carolina Gonzálvez enjoyed a research-leave from the University of Alicante, where she is located. This article is the result of the research work initiated and developed to a relevant stage during that period: Definition of the research question, selection criteria and first analysis of results.

\section{References $^{2}$}

Achenbach, T. M., \& Rescorla, L. (2003). Manual for the ASEBA adult forms \& profiles. University of Vermont, Research Center for Children, Youth, and Families.

Alonso, J., Prieto, L., \& Antó, J. M. (1995). La versión española del SF-36 health survey (Cuestionario de Salud SF-36): Un instrumento para la medida de los resultados clínicos. Medicina Clínica, 104(20), 771-776.

Alson, J. N., Espela, C. S., \& Urbina, M. A. C. O. (2019). Factors affecting employability of persons with disabilities. International Journal of Multidisciplinary Research and Publications, 1(9), 1-5.

Appleton, J. J., Christenson, S. L., Kim, D., \& Reschly, A. L. (2006). Measuring cognitive and psychological engagement: Validation of the student engagement instrument. Journal of School Psychology, 44(5), 427-445. https://doi.org/10.1016/j.jsp.2006.04.002

Badiola, V., Barroso, J., Cornejo, I., De La Serna, F., Elorriaga, E., Etxebarria, J., Franco, T., Igeregi, I., Iturbide, P., Martín, F., Martín, M., Martínez, F. J., Mata, B., Mercado, A., Molinos, A., Navarro, S.,

2 References preceded by an asterisk were included in the meta-analysis. 
Restrepo, W., Santamaría, T., \& Zulueta, B. (2014). Método de Perfiles LantegiBatuak de adecuación de la tarea a la persona. Lantegi Batuak Fundazioa.

Barlow, J., Wright, C., \& Cullen, L. (2002). A job-seeking self-efficacy scale for people with physical disabilities: Preliminary development and psychometric testing. British Journal of Guidance \& Counseling, 30(1), 37-53. https://doi.org/10.1080/030698880220106500

Becker, H. A. (1980). The assertive job-hunting survey. Measurement and Evaluation in Guidance, 13(1), 43-48. https://doi.org/10.1080/00256307.1980.12022216.

Bell, M., Bryson, G., \& Lysaker, P. (1997). Positive and negative affect recognition in schizophrenia: A comparison with substance abuse and normal control subjects. Psychiatry Research, 73, 73-82. https://doi.org/10.1016/s0165-1781(97)00111-x

Bengoetxea, A., \& Fajardo, G. (2020). La inclusión socio-laboral de colectivos vulnerables en las empresas de economía social. CIRIEC-España, Revista Jurídica de Economía Social y Cooperativa, 36, 9-12.

Borenstein, M., Hedges, L.V., Higgins, J. P. T., \& Rothstein, H. R. (2010). A basic introduction to fixedeffect and random-effects models for meta-analysis. Research Synthesis Methods, 1, 97-111. https:// doi.org/10.1002/jrsm.12

Botella, J., \& Sánchez-Meca, J. (2015). Meta-análisis en ciencias sociales y de la salud. Síntesis.

Brown, L., \& Leigh, J. E. (1986). Adaptive Behavior Inventory. Pro-Ed.

Bundock, K. E., \& Hewitt, O. (2017). A review of social skills interventions for adults with autism and intellectual disability. Tizard Learning Disability Review, 22(3), 148-158. https://doi.org/10.1108/ TLDR-05-2016-0015

Carter, E. W., Lane, K. L., Cooney, M., Weir, K., Moss, C. K., \& Machalicek, W. (2013). Self-determination among transition-age youth with autism or intellectual disability: Parent perspectives. Research and Practice for Persons with Severe Disabilities, 38(3), 129-138. https://doi. org/10.1177/154079691303800301

Casal, J. (1996). Modos emergentes de transición a la vida adulta en el umbral del siglo XXI: Aproximación sucesiva, precariedad y desestructuración. Revista española de investigaciones sociológicas, $75,295-318$.

Casal, J., García, M., \& Merino, R. (2015). Pasado, presente y future de los estudios sobre las transiciones de los jóvenes. Revista de Estudios de Juventud, 110, 69-81.

Cavadel, E. W., Kauff, J. F., Anderson, M. A., McConnell, S., \& Derr, M. (2017). Self-regulation and goal attainment: A new perspective for employment programs. Office of Planning, Research, and Evaluation, Administration for Children and Families U.S. Department of Health and Human Services.

Centers for Disease Control and Prevention. (CDC) (2018). YRBSS - Youth risk behavior surveillance system: Data - Adolescent and school health. https://www.cdc.gov/healthyyouth/data/yrbs/index. htm

Chesney, M. A., Neilands, T. B., Chambers, D. B., Taylor, J. M., \& Folkman, S. (2006). A validity and reliability study of the coping self-efficacy scale. British Journal of Health Psychology, 11(3), 421-437. https://doi.org/10.1348/135910705X53155

Cheung, M. W.-L. (2014). Modeling dependent effect sizes with three-level meta-analyses: A structural equation modeling approach. Psychological Methods, 19(2), 211-229. https://doi.org/10.1037/ a0032968

Cheung, M. W.-L. (2019). A guide to conducting a meta-analysis with non-independent effect sizes. Neuropsychology Review, 29, 387-396. https://doi.org/10.1007/s11065-019-09415-6 
Chisvert, M. J., Palomares, D., Hernáiz, N., \& Salinas, M. (2018). Trayectorias de aprendizaje en contextos informales. El caso de tres empresas de inserción. Ciriec, 94, 185-216. http://ciriec-revistaeconomia.es/wp-content/uploads/CIRIEC_9407_Chisvert_et_al.pdf

Colón, H., \& Haertlein, C. (2002). Spanish translation of the role checklist. American Journal of Occupational Therapy, 56(5), 586-589. https://doi.org/10.5014/ajot.56.5.586

Constantino, J. N., \& Gruber, C. P. (2005). Social responsiveness scale (SRS). Western Psychological Services.

Córdoba, A., Llinares, L., \& Zacarés, J. J. (2013). Employability assessment in vocational education and transition to the workplace. In J. Seifried \& E. Wuttke (Eds.), Transitions in vocational education, research in vocational education (pp. 181-200). Budrich.

Cummins, R., \& Lau, A. (2005). Personal wellbeing index-school children (PWI-SC) (3rd ed.). Deakin University.

Douvitsa, I. (2020). The work integration social enterprises in Greece. CIRIEC-España, Revista Jurídica de Economía Social y Cooperativa, 36, 195-219. https://doi.org/10.7203/CIRIEC-JUR.36.17289

Dyke, P., Bourke, J., Llewellyn, G., \& Leonard, H. (2013). The experiences of mothers of young adults with an intellectual disability transitioning from secondary school to adult life. Journal of Intellectual \& Developmental Disability, 38(2), 149-162. https://doi.org/10.3109/13668250.2013.789099

Elorriaga, E., Restrepo, W., \& Zulueta, B. (2019). Modelo de apoyos individuales en Lantegi Batuak. Manual práctico. Lantegi Batuak Fundazioa.

Engelbrecht, M., Shaw, L., \& Van Niekerk, L. (2017). A literature review on work transitioning of youth with disabilities into competitive employment. African Journal of Disability, 6(298), 1-7. https:// doi.org/10.4102/ajod.v6i0.298

Eraut, M. (2004). Informal learning in the workplace. Studies in continuing education, 26(2), 247-273. https://doi.org/10.1080/158037042000225245

Eurofound. (2013). Impact of the crisis on working conditions in Europe. European Foundation for the Improvement of Living and Working Conditions.

European Union. (2010). European disability strategy 2010-2020: A renewed commitment to a barrierfree Europe. European Union.

Farley, R. (1988). Vocational self-awareness scale. University of Arkansas Research and Training Center in Vocational Rehabilitation.

${ }^{\star}$ Fernández-Solano, A. J., Del Baño-Aledo, M. E., \& Rodríguez-Bailón, M. (2019). From thinking to acting: Occupational self-analysis tools for use with people with intellectual disability. A pilot study. Journal of Intellectual Disability Research, 63(9), 1086-1096. https://doi.org/10.1111/jir.12621

Fici, A. (2020). La empresa social italiana después de la reforma del tercer sector. CIRIEC-España, Revista Jurídica de Economía Social y Cooperativa, 36, 177-193. https://doi.org/10.7203/CIRIECJUR.36.17109

Glass, G. V., McGaw, B., \& Smith, M. L. (1981). Meta-analysis in social research. SAGE.

Gilson, C. N., Carter, E. W., \& Biggs, E. E. (2017). Systematic review of instructional methods to teach employment skills to secondary students with intellectual and developmental disabilities. Research and Practice for Persons with Severe Disabilities, 42(2), 89-107. https://doi. org/10.1177/1540796917698831

Goldman, B. M., Gutek, B. A., Stein, J. H., \& Lewis, K. (2006). Employment discrimination in organizations: Antecedents and consequences. Journal of Management, 32, 786-830. https://doi. org/10.1177/0149206306293544 
Gonon, P., Kraus, K., Oelkers, J., \& Stolz, S. (2008). Work, education and employability. Peter Lang. Gresham, F. M., \& Elliott, S. N. (1990). Social skills rating system (SSRS). American Guidance.

Harrison, P., \& Oakland. T. (2015). Adaptive behavior assessment system (3rd ed.). Western Psychological Services.

${ }^{*}$ Hatfield, M., Falkmer, M., Falkmer, T., \& Ciccarelli, M. (2017). Effectiveness of the BOOST-A ${ }^{\mathrm{m}}$ online transition planning program for adolescents on the autism spectrum: A quasi-randomized controlled trial. Child and Adolescent Psychiatry and Mental Health, 11(54), 1-12. https://doi. org/10.1186/s13034-017-0191-2

${ }^{*}$ Hayes, G. R., Custodio, V. E., Haimson, O. L., Nguyen, K., Ringland, K. E., Ulgado, R. R., Waterhouse, A., \& Weiner, R. (2015). Mobile video modeling for employment interviews for individuals with autism. Journal of Vocational Rehabilitation, 43, 275-287. https://doi.org/10.3233/JVR-150775

Hedges, L., \& Olkin, I. (1985). Statistical methods for meta-analysis. Harcourt, Brace, \& Jovanovich.

Hedley, D., Uljarevic, M., Cameron, L., Halder, S., Richdale, A., \& Dissanayake, C. (2017). Employment programmes and interventions targeting adults with autism spectrum disorder: A systematic review of the literature. Autism, 21(8), 929-941. https://doi.org/10.1177/1362361316661855

Higgins, J. P. T., \& Thompson, S. G. (2002). Quantifying heterogeneity in a meta-analysis. Statistics in Medicine, 21, 1539-1558. https://doi.org/10.1002/sim.1186

Hofmann, C., Häfeli, K., Müller, X., \& Krauss, A. (2021). Transition from low-threshold vocational education and training to work in Switzerland: Factors influencing objective and subjective career success. International Journal for Research in Vocational Education and Training, 8(2), 136-159. https://doi.org/10.13152/IJRVET.8.2.1

Holland, J. L, Daiger, D. C., \& Power, P. G. (1980). My vocational situation. Consulting Psychologists Press.

Ibáñez, P., \& Mudarra, M. J. (2005). Relaciones sociales en el empleo, en trabajadores con discapacidad. Educación XXI, 8, 105-125.

International Labour Organization. (ILO) (2016). Including persons with disabilities in Technical and Vocational education and training. A guide for administrators and instructors to disability inclusion. International Labour Organization.

${ }^{\star}$ Ipsen, C., Kurth, N., McComick, S., Hall, J., \& Chambless, C. (2019). Exploring the PROMISE of transition services for youth with disabilities receiving SSI. Journal of Vocational Rehabilitation, 50, 95-108. https://doi.org/10.3233/JVR-180991

Jordan, S., Gessnitzer, S., \& Kauffeld, S. (2016). Effects of a group coaching for the vocational orientation of secondary school pupils. Coaching: An International Journal of Theory, Research and Practice, 9(2), 143-157. https://doi.org/10.1080/17521882.2016.1210185

Koren, P., DeChillo, N., \& Friesen, B. (1992). Measuring empowerment in families whose children have emotional disabilities: A brief questionnaire. Rehabilitation Psychology, 37(4), 305-321. https://doi.org/10.1037/h0079106

${ }^{\star}$ Kumakazi, H., Warren, Z., Corbett, B. A., Yoshikawa, Y., Matsumoto, Y., Higashida, H., Yuhi, T., Ikeda, T., Ishiguro, H., \& Kikuchi, M. (2017). Android robot-mediated mock job interview sessions for young adults with autism spectrum disorder: A pilot study. Frontiers in Psychology, 8, 169. https://doi.org/10.3389/fpsyt.2017.00169

Lee, G. K., Chun, J., Hama, H., \& Carter, E. W. (2018). Review of transition and vocational interventions for youth and adults with autism spectrum disorders. Review Journal of Autism and Developmental Disorders, 5, 268-284. https://doi.org/10.1007/s40489-018-0138-4. 
${ }^{\star}$ Lindstrom, L., Doren, B., Post, C., \& Lombardi, A. (2013). Building career PATHS (Postschool Achievement Through Higher Skills) for young women with disabilities. The Career Development Quarterly, 61, 330-338. https://doi.org/10.1002/j.2161-0045.2013.00059.x

Lipsey, M. W. (1994). Identifying potentially interesting variables and analysis opportunities. In $\mathrm{H}$. Cooper \& L. V. Hedges (Eds.), The handbook of research synthesis (pp. 111-123). Russel Sage Foundation.

Lipsey, M. W. (2009). Identifying interesting variables and analysis opportunities. In H. M. Cooper, L. V. Hedges \& J. C. Valentine (Eds.), The handbook of research synthesis and meta-analysis (pp. 147-158). SAGE.

Llinares L., Córdoba, A. I., \& González-Navarro, P. (2020a). La empleabilidad a debate: ¿qué sabemos sobre la empleabilidad como estrategia de cambio social? CIRIEC-España, Revista Jurídica de Economía Social y Cooperativa, 36, 313-363. https://doi.org/10.7203/CIRIEC-JUR.36.17017

Llinares L., Córdoba, A. I., Zacarés, J. J., \& González-Navarro, P. (2020b). Indicadores de empleabilidad: De la inclusión al desarrollo de las carreras laborales. CIRIEC-España, Revista Jurídica de Economía Social y Cooperativa, 36, 365-387. https://doi.org/10.7203/CIRIEC-JUR.36.17016

Llinares, L., Zacarés, J. J., \& Córdoba, A. I. (2016). Discussing employability: Current perspectives and key elements from a bioecological model. Employee Relations, 38(6), 961-974. https://doi. org/10.1108/ER-07-2015-0145

Lombardi, A., Gerdes, H., \& Murray, C. (2011). Validating an assessment of individual actions, postsecondary supports, and social supports of college students with disabilities. Journal of Student Affairs Research and Practice, 48, 107-126. https://doi.org/10.2202/1949-6605.6214

López-Bastías, J. L. (2019). La conceptualización de la discapacidad a través de la historia: Una mirada a través de la evolución de la normativa. Revista de la Facultad de Derecho de México, 69(273), 835-855. https://doi.org/10.22201/fder.24488933e.2019.273-2.68632

Madans, J., Loeb, M., \& Eide, A. H. (2017). Measuring disability and inclusion in relation to the 2030 Agenda on Sustainable Development. Disability and the Global South, 4(1), 1164-1179.

Maffiioletti, E. U., \& Sato, C. (2020). A inserçao social e laboral de grupos vulneráveis nos empreendimentos sociais no Brasil por meio das cooperativas. CIRIEC-España, Revista Jurídica de Economía Social y Cooperativa, 36, 249-272. https://doi.org/10.7203/CIRIEC-JUR.36.17168

Marhuenda, F. (2018). Learning at work: Researching personal development and competence building in work integration companies. Educar, 54(2), 491-509.

Marhuenda, F. (2021). Apprenticeships for adulthood. The contribution of the third sector to nonformal vocational education. In P. Eigenmann, P. Gonon \& M. Weil (Eds.), Opening and extending vocational Education (pp. 291-318). Peter Lang.

Martin, J., \& Marshall, L. (1993). The self-directed IEP. University of Colorado at Colorado Springs.

Martínez-Ríos, B. (2011). Pobreza, Discapacidad y Derechos Humanos. Aproximación a los costes extraordinarios de la discapacidad y su contribución a la pobreza desde un enfoque basado en los derechos humanos. Ediciones Cinca.

${ }^{*}$ Mawhood, L., \& Howlin, P. (1999). The outcome of a supported employment scheme for highfunctioning adults with autism or asperger syndrome. Autism, 3(3), 229-254. https://doi. org/10.1177/1362361399003003003

McInnes, M. D. F., Moher, D., Thombs, B. D., McGrath, T. A., Bossuyt, P. M., Clifford, T., Cohen, J. F. et al. (2018). Preferred reporting items for a systematic review and meta-analysis of diagnostic test 
accuracy studies: The PRISMA-DTA statement. JAMA, 319(4), 388-396. https://doi.org/10.1001/ jama.2017.19163

McWhirter, E. H., Rasheed, S., \& Crothers, M. (2000). The effects of high school career education on social-cognitive variables. Journal of Counseling Psychology, 47(3), 330-341. https://doi. org/10.1037/0022-0167.47.3.330

Meira, D. (2020). O fin mutualístico desiteressado our altruísta das cooperativas de solidariedade social. CIRIEC-España, Revista Jurídica de Economía Social y Cooperativa, 36, 221-247. https://doi. org/10.7203/CIRIEC-JUR.36.17386

Merino, R. (2007). Pathways from school to work: Can the competence acquired in leisure activities improve the construction of pathways? Journal of education and work, 20(2), 139-159. https://doi. org/10.1080/13639080701314696

Merino, R. (2019). Los componentes no formales de los itinerarios formativos de los jóvenes, ¿refuerzan o compensan las desigualdades sociales en educación? Revista de estudios de juventud, $124,209-226$

Metheny, J., McWhirter, E. H., \& O'Neil, M. E. (2008). Measuring perceived teacher support and its influence on adolescent career development. Journal of Career Assessment, 16, 218-237. https://doi. org/10.1177/1069072707313198

Morelock, K., Roessler, R., \& Bolton, B. (1987). The employability maturity interview: Reliability and construct validity. Vocational Evaluation \& Work Adjustment Bulletin, 20(2), 53-59.

${ }^{*}$ Murray, C., \& Doren, B. (2013). The effects of working at gaining employment skills on the social and vocational skills of adolescents with disabilities: A school-based intervention. Rehabilitation Counseling Bulletin, 56(2), 96-107. https://doi.org/10.1177/0034355212452614

National Institute of Stastistics. (2019). El empleo de las personas con discapacidad 2017. National Institute of Stastistics.

National Institute of Stastistics. (2017). El salario de las personas con discapacidad 2014. National Institute of Stastistics.

Novo-Corti, I. (2018). La inclusión efectiva de las personas con discapacidad en un entorno laboral normalizado: Dificultades y retos. Panorama Social, 26, 79-92.

Offer, D., Ostrov, E., Howard, K. I., \& Dolan, S. (1992). Offer self-image questionnaire. Revised. Western Psychological Services.

*O'Mally, J., \& Antonelli, K. (2016). The effect of career mentoring on employment outcomes for college students who are legally blind. Journal of Visual Impariment \& Blindness, 110(5), 295-307. https://doi.org/10.1177/0145482X1611000502

${ }^{\star}$ Oswald, T. M., Winder-Patel, B., Ruder, S., Xing, G., Stahmer, A., \& Solomon, M. (2017). A pilot randomized controlled trial of the ACCESS Program: A group intervention to improve social, adaptive functioning, stress coping, and self-determination outcomes in young adults with autism spectrum disorder. Journal of Autism and Developmental Disorders, 48(5), 1742-1760. https://doi. org/10.1007/s10803-017-3421-9

Pallisera, M., Vilà, M., Fullana, J., Castro, M., Puyaltó, C., \& Díaz-Garolera, G. (2017). Proposals for improving the transition process of young people with intellectual disabilities in Spain: Insights from focus groups of professionals, young people and their families. European Journal of Special Needs Education, 33(3), 1-15. https://doi.org/10.1080/08856257.2017.1306966 
Park, E., Kim, J., \& Kim, S. (2016). Meta-analysis of the effect of job-related social skill training for secondary students with disabilities. Journal of Vocational Rehabilitation, 44, 123-133. https://doi. org/10.3233/JVR-150785

${ }^{\star}$ Powers, L. E., Turner, A., Westwood, D., Matuszewski, J., Wilson, R., \& Phillips, A. (2001). TAKE CHARGE for the future: A controlled field-test of a model to promote student involvement in transition planning. Career Development and Transition for Exceptional Individuals, 24(1), 89-104. https://doi.org/10.1177/088572880102400107

Randolph, C., Tierney, M. C., Mohr, E., \& Chase, T. N. (1998). The repeatable battery for the assessment of neuropsychologicalstatus (RBANS): Preliminary clinical validity. Journal of Clinical and Experimental Neuropsychology, 20, 310-319. https://doi.org/10.1076/jcen.20.3.310.823

Rodríguez-Bailón, M., Pulido-Navarro, M. J., Bravo-Quirós, A., Alberto-Cantizano, M. J., RubioFernández, V., \& Fernández-Solano, A. J. (2016). Seeing, judging and ...acting! Improving health perception and meaningful occupational participation in older Spanish adults. Educational Gerontology, 42, 572-584. https://doi.org/10.1080/03601277.2016.1205384

Review Manager (RevMan) [Computer program]. (2014). Version 5.3. The Cochrane Collaboration.

Rosenberg, M. (1965). Society and the adolescent self-image. Princeton University Press.

Rottinghaus, P. J., Day, S. X., \& Borgen, F. H. (2005). The career futures inventory: A measure of career-related adaptability and optimism. Journal of Career Assessment, 13(1), 3-24. https://doi. org/10.1177/1069072704270271

Salinas, M. F., \& Marhuenda, F. (2020). Acceso al empleo de las personas con discapacidad: Situación en el empleo en las personas con enfermedad mental. CIRIEC-España, Revista Jurídica de Economía Social y Cooperativa, 36, 273-311. https://doi.org/10.7203/CIRIEC-JUR.36.16980

Sánchez-Meca, J., \& Botella, J. (2010). Revisiones sistemáticas y meta-análisis: Herramientas para la práctica profesional. Papeles del Psicólogo, 31(1), 7-17.

Sandoya, E. (2008). Metaanálisis: Fortalezas y debilidades. Revista Uruguaya de Cardiología, 23(3), $263-275$.

Schur, L., Kruse, D., Blasi, J., \& Blanck, P. (2009). Is disability disabling in all workplaces? Workplace disparities and corporate culture. Industrial Relations, 48, 381-410. https://doi.org/10.1111/j.1468232X.2009.00565.x

Senent-Ramos, M. (2020). Functional diversity or disability? Social and conceptual challenges of the new educational paradigms. Culture and Education, 32(1), 17-26. https://doi.org/10.1080/113564 05.2019.1709397

${ }^{\star}$ Smith, M. J., Ginger, E., Wright, K., Wright, M. A., Taylor, J. L., Humm, L. B., Olsen, D. E., Bell, M., \& Fleming, M. F. (2014). Virtual reality job interview training in adults with autism spectrum disorder. Journal of Autism and Developmental Disorders, 44, 2450-2463. https://doi.org/10.1007/ s10803-014-2113-y

*Strickland, D. C., Coles, C. D., \& Sothern, L. B. (2013). JobTIPS: A transition to employment program for individuals with autism spectrum disorders. Journal of Autism and Developmental Disorders, 43, 2472-2483. https://doi.org/10.1007/s10803-013-1800-4

Taylor, J. L., McPheeters, M. L., Sathe, N. A., Dove, D., Veenstra-VanderWeele, J., \& Warren, Z. (2012). A systematic review of vocational interventions for young adults with autism spectrum disorders. Pediatrics, 130(3), 531-538. https://doi.org/10.1542/peds.2012-0682

Thompson, A., Lindeman, R., Super, D., Jordaan, J., \& Myers, R. (1981). Career development inventory: User's manual. Consulting Psychologists Press. 
United Nations. (2006). Convention on the rights of persons with disabilities. United Nations. https:// www.un.org/disabilities/documents/convention/convoptprot-e.pdf

${ }^{\star}$ Verdugo, M. A., Jenaro, C., \& Arias, B. (1998). Outcomes of a program of vocational evaluation for persons with physical disabilities and deafness. Journal of Vocational Rehabilitation, 11(3), 181194. https://doi.org/10.3233/JVR-1998-11304

Wakabayashi, A., Tojo, Y., Baron-Cohen, S., \& Wheelwright, S. (2004). The autism spectrum quotient (AQ) Japanese version: Evidence from high-functioning clinical group and normal adults. Shinrigaku Kenkyu, 75, 78-84. https://doi.org/10.4992/jjpsy.75.78

Walsh, E., Holloway, J., McCoy, A., \& Lydon, H. (2017). Technology-aided interventions for employment skills in adults with autism spectrum disorder: A systematic review. Review Journal of Autism and Developmental Disorders, 4(1), 12-25. https://doi.org/10.1007/s40489-016-0093-x

Walther, A. (2006). Regimes of youth transitions. Young, 14(2), 119-139. https://doi. org/10.1177/1103308806062737

Wehmeyer, M. L., \& Kelchner, K. (1995). The arc's self-determination scale. Arc.

Williams, G., \& Deci, E. (1996). Internalization of biopsychosocial values by medical students: A test of self-determination theory. Journal of Personality and Social Psychology, 70(4), 767-779. https:// doi.org/10.1037/0022-3514.70.4.767

Wolman J., Campeau, P., Dubois, P., Mithaug, D., \& Stolarski, V. (1994). AIR self-determination scale and user guide. American Institute on Research.

\section{Biographical Notes}

Carolina Gonzálvez, Professor at the Department of Developmental Psychology and Teaching of the Faculty of Education at the University of Alicante, Spain. Her main line of research focuses on the evaluation of school attendance problems and people with disability. She is co-author in more than 80 articles, she has contributed with more than 180 proposals in national and international congresses, and has collaborated as a member of different scientific committees. She has participated in groups and research projects funded by public institutions and she is the head researcher of the Projects GRE16-07 and GV/2019/075 about school refusal.

Fernando Marhuenda-Fluixá, Professor at the Universitat de València, coordinator of the research group Transicions, winner of the 2019 European VETskills Research Award European Comission: An interdisciplinary team with a focus on the intersection between vocational education, adult education and social inclusion. Member of the Board of the EERA-VETNET Network he has published Vocational Education beyond Skill Formation: VET between Civic, Industrial and Market Tensions (Peter Lang, 2017), The School-Based Vocational Education and Training System in Spain: Achievements and Controversies (Springer, 2019) and coedited Apprenticeships in dual and non-dual systems (Peter Lang, 2021). 